\title{
TRIGONOMETRIA ESFÉRICA APLICADA NA ASTRONOMIA DE POSIÇÃO
}

\section{Applied spherical trigonometry in position astronomy}

\author{
Christian Luz Pelissari de Oliveira ${ }^{1}$; Fernando Pereira de Souza ${ }^{2}$ \\ ${ }^{1}$ Universidade Federal de Mato Grosso do Sul \\ Departamento de Ciências Exatas \\ Av. Ranulpho Marques Leal, 3484, Três Lagoas - MS, 79610-100 - Brasil \\ christianluz09@gmail.com
}

\author{
${ }^{2}$ Universidade Federal de Mato Grosso do Sul \\ Departamento de Ciências Exatas \\ Av. Ranulpho Marques Leal, 3484 , Três Lagoas - MS, 79610-100 - Brasil \\ fermatmel@gmail.com
}

RESUMO - O presente artigo é resultado de um trabalho de pesquisa do Curso de Licenciatura em Matemática no âmbito do Programa de Educação Tutorial PET. O trabalho aborda conceitos da Trigonometria Esférica, que possui diversos campos de aplicações entre a matemática e a física, relacionados em problemas cartográficos, na navegação e na astronomia. O objetivo é explorar problemas de aplicações da astronomia dos corpos celestes, fazendo o uso de conceitos de trigonometria na esfera para estudar posições e direções de astros em termos de uma esfera celeste. Para atingir tal objetivo, o artigo apresenta conceitos de menor distância entre dois pontos na esfera, triângulo de posição que é o triângulo esférico, a relação fundamental conhecida como lei dos cossenos, a Esfera Celeste, seus elementos, as suas coordenadas no sistema equatorial, sistema horizontal e sistema horário. Assim, o trabalho busca incentivar alunos e professores a trabalharem a Geometria Esférica na sala de aula.

Palavras-chave: geometria esférica; esfera celeste; astronomia de posição.

Recebido em: 24/04/2018 Revisado em: 24/08/2018 Aprovado em: 13/09/2018
ABSTRACT - The present article is the result of a research work of the Degree in Mathematics in the scope of the Tutorial Education Program - PET. The work deals with concepts of Spherical Trigonometry, which has several fields of applications between mathematics and physics, related to cartographic problems, navigation and astronomy. The goal is to explore problems of astronomy applications of celestial bodies by making use of trigonometry concepts in the sphere to study positions and directions of stars in terms of a celestial sphere. In order to reach this objective, the article presents concepts of a smaller distance between two points in the sphere, a triangle of position that is the spherical triangle, the fundamental relation known as law of cosines, the Celestial Sphere, its elements, its coordinates in the equatorial system, horizontal system and time system. Thus, the work seeks to encourage students and teachers to work on Spherical Geometry in the classroom. Keywords: spherical geometry; celestial sphere; position astronomy. 


\section{INTRODUÇÃO}

Como sabemos, a forma do planeta Terra em comparação com uma forma geométrica se aproxima de uma esfera. Alguns conceitos da geometria esférica são vistos no decorrer da educação básica, situações que são vistas nas aulas de geografia como, linha do Equador, meridianos, paralelos, latitudes e longitudes. Os conceitos geométricos da esfera tais como, o volume, a superfície, o fuso e a área são vistos nas aulas de matemática. Algumas observações devem estar subentendidas perante o aluno no fim de sua formação diante da geometria, onde os conceitos da geometria plana não são validos na geometria esférica, por exemplo, qual é a menor distância entre dois pontos na geometria esférica? Sendo que na Euclidiana seria o segmento de reta, mas isso existindo apenas na Geometria Plana, neste caso se faz comparações, com que os pontos se tornam cidades próximas e a menor distância seria tal estrada, sendo um arco com a circunferência máxima.

O objetivo do presente trabalho se da em explorar aplicações astronômicas com estudos na geometria esférica e trigonométrica, seus aspectos espaciais, geográficos. Fazendo um estudo analítico de seu corpo. Consequentemente, abordando e aplicando problemas da astronomia de posição.

\section{METODOLOGIA}

O trabalho foi desenvolvido através de estudo teórico, prático e pesquisa bibliográfica, questões teóricas da astronomia, demonstrações e análise de teoremas sendo aplicadas na geometria esférica, ambas estudadas na forma de apresentação de seminários, resolução de exercícios, interpretações geométricas e trigonométricas das aplicações astronômicas de posição.

\section{RESULTADOS}

Dado ao estudo fundamental da trigonometria esférica, demonstraremos a lei dos cossenos na esfera.

Definição 1: O plano que faz uma secção em uma esfera é chamado de plano secante. Se esse plano secante passa pelo centro da esfera, a circunferência formada na secção é chamada de circunferência máxima.

Figura 1: Circunferência Máxima



Fonte: O Autor

Os meridianos e o equador são exemplos de círculos máximos traçados sobre a superfície da Terra sendo que os círculos paralelos que cruzam com os meridianos originários da rotação do planeta, são gradativamente menores a partir que se afastam da equador em direção aos pólos.

Definição 2: Ângulo esférico: é o ângulo, formado por dois arcos de circunferências máximas. Sua medida é a mesma do ângulo plano formado pelas semirretas tangentes a esses arcos. 
Figura 2: Ângulo Esférico

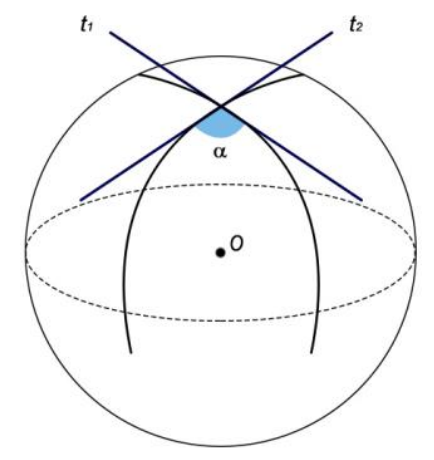

Fonte: O Autor

Definição 3: Triângulo esférico é uma superfície limitada por três arcos circunferências máximas, contida em algum hemisfério, sendo estes arcos menores que uma semicircunferência máxima.

Figura 3: Triângulo Esférico

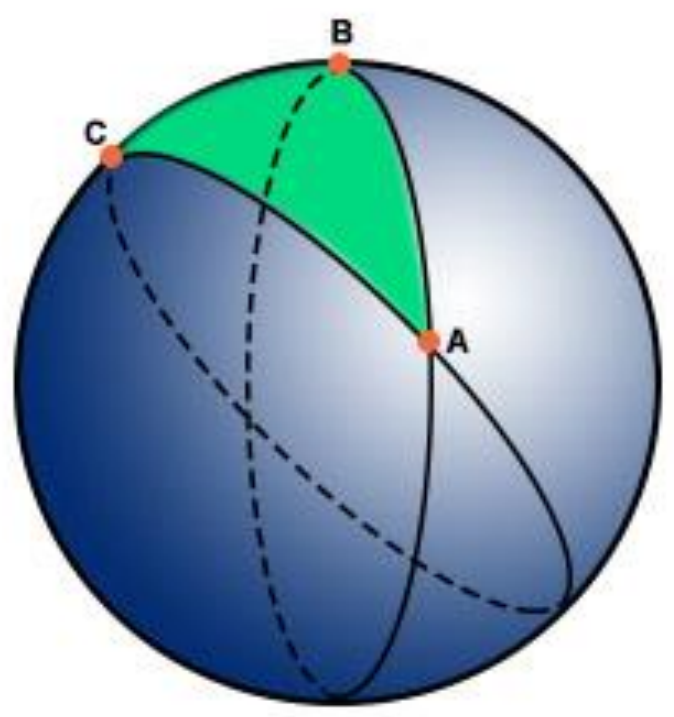

Fonte: O Autor

Teorema 1: Teorema fundamental do triângulo esférico. Seja $A B C$ um triângulo esférico, com lados $a, b$ e $c$, e ângulos internos $A, B$ e $C$. Então

$$
\begin{aligned}
& \cos (a)=\cos (b) \cos (c) \\
& \quad+\operatorname{sen}(b) \operatorname{sen}(c) \cos A(1) \\
& \cos (b)=\cos (a) \cos (c) \\
& \quad+\operatorname{sen}(a) \operatorname{sen}(c) \cos B(2)
\end{aligned}
$$

$$
\begin{aligned}
\cos (c)= & \cos (a) \cos (b) \\
& +\operatorname{sen}(a) \operatorname{sen}(b) \cos C
\end{aligned}
$$

Dem: Seja $r$ a reta tangente em $A$ ao arco $O B$ e $S$ a reta tangente em $A$ ao arco $A C$. Desta forma a reta $r$ intersecta a reta $O B$ em um ponto que chamaremos de $D$, da mesma forma, a reta $s$ intersecta a reta $O C$ em um ponto que chamaremos de $E$.

Figura 4: Teorema Fundamental



Fonte: O Autor

Observe que as retas $r$ e $s$ são tangentes à esfera, contudo $A O$ e $A D$ são perpendiculares, isso sendo análogo para $O A$ e $A E$.

Analisando os triângulos $O D A$ e $O E A$, da geometria euclidiana temos:

$$
\begin{aligned}
& \cos (b)=\frac{\overline{A O}}{\overline{\frac{O E}{A O}}}, \\
& \cos (c)=\frac{\overline{A O}}{\overline{\frac{O D}{A E}}}, \\
& \operatorname{sen}(b)=\frac{\overline{\overline{O E}}}{\operatorname{AD}} \\
& \operatorname{sen}(c)=\frac{\overline{O D}}{\overline{O D}} .
\end{aligned}
$$

Desta maneira, aplicando teorema de Pitágoras em ambos os triângulos, obtemos:

$$
\overline{O D}^{2}=\overline{A D}^{2}+\overline{O A}^{2}
$$

e

$$
\overline{O E}^{2}=\overline{A E}^{2}+\overline{O A}^{2},
$$

somando os resultados, teremos:

$$
\overline{O D}^{2}+\overline{O E}^{2}=\overline{A D}^{2}+2 \overline{O A}^{2}+\overline{A E}^{2} .
$$


Aplicando a lei dos cossenos (geometria plana) nos triângulos $A D E$ e $O D E$ e igualando temos:

$$
\begin{aligned}
\overline{O D}^{2}+\overline{O E}^{2}- & 2 \overline{O D} \cdot \overline{O E} \cdot \cos (a) \\
& =\overline{A D}^{2} \cdot \overline{A E}^{2}-2 \overline{A D} \cdot \overline{A E} \\
& \cdot \cos A
\end{aligned}
$$

assim, de (5), obtemos:

$$
\cos (a)=\frac{\overline{A O}^{2}}{\overline{O D} \cdot \overline{O E}}+\frac{\overline{A D} \cdot \overline{A E}}{\overline{O D} \cdot \overline{O E}} \cdot \cos A,
$$

Portanto, usando (4), concluímos que:

$$
\begin{aligned}
\cos (a)=\cos (b) \cos (c) \\
\\
+\operatorname{sen}(b) \operatorname{sen}(c) \cos A .
\end{aligned}
$$

As equações (2) e (3) são análogas (ABREU,2015).

\subsection{A Esfera Celeste}

Para o estudo da geometria esférica se faz necessário o entendimento de alguns conceitos da Astronomia de posição, segundo (ABREU,2015).

Zênite: é o termo técnico que designa o ponto (imaginário) interceptado por um eixo vertical (imaginário) traçado a partir da cabeça de um observador (localizado sobre a superfície terrestre) e que se prolonga até a esfera celeste.

Nadir: ponto da esfera celeste oposto ao zênite, que se situa na vertical do observador, diretamente sob seus pés.

Polo Celeste Norte: ponto em que o prolongamento do eixo de rotação da Terra intercepta a esfera celeste, no hemisfério norte.

Polo Celeste Sul: ponto em que o prolongamento do eixo de rotação da Terra intercepta a esfera celeste, no hemisfério sul.
Figura 5. Conceitos da Astronomia de Posição

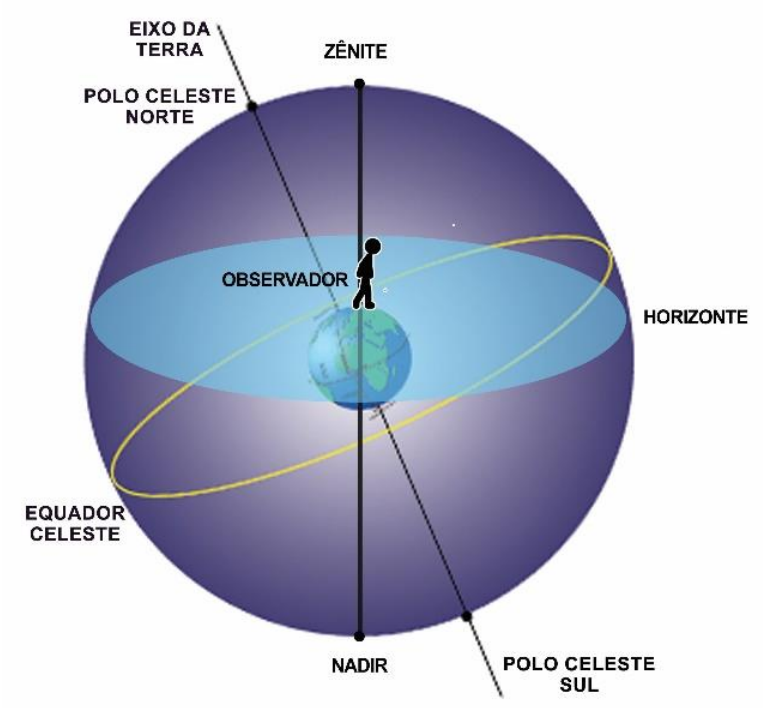

Fonte: O Autor

\subsubsection{Sistema de Coordenada Horizontais}

Sistema geocêntrico: origem no centro da Terra;

Orientação: levogiro ou retrógrado;

Plano fundamental: horizonte astronômico do observador;

Coordenadas:

$(A)=$ azimute (abscissa esférica)

(h): altura

(z): distância zenital (ordenada)

Eixos:

$X$ : formado pela interseção do meridiano do observador com o horizonte;

$Y$ : coincide com a linha Leste - Oeste;

$Z$ : coincide com a vertical do observador.

Figura 6. Sistema de Coordenadas Horizontais

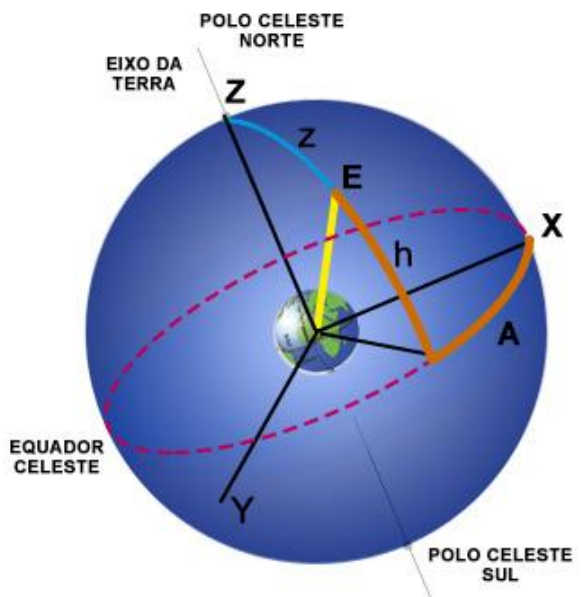

Fonte: O Autor 
Neste sistema de coordenadas, temse: a abscissa esférica é o azimute, definido como o ângulo contado sobre o horizonte, deste o ponto sul, por oeste (sentido retrógrado), até a vertical que contém o astro. Varia de $0^{\circ} a 360^{\circ}$. Quando o astro "cruza" o meridiano, seu azimute será $0^{\circ}$ ou $180^{\circ}$ (ARANA, 2000).

A ordenada esférica é a altura, ângulo contado desde o horizonte, sobre a vertical do astro, até o astro. Varia de $0^{\circ}$ (astro no horizonte, nascendo ou ocultando) $a \pm 90^{\circ}$ (astro no zênite ou no nadir), alturas negativas correspondem a astros situados abaixo do horizonte, e portanto, invisível ao observador.

Em muitos problemas é conveniente, ao invés de se usar altura, utiliza-se a distância zenital $Z$, que é o ângulo contado a partir do zênite até o astro sobre o seu vertical, varia de $0^{\circ}$ (astro no zênite) a $180^{\circ}$ (astro no nadir).

Astro no horizonte tem-se:

Tem-se que:

$$
h=0^{\circ} \text { ou } Z=90^{\circ} \text {. }
$$

$$
Z=90^{\circ}-h
$$

Neste sistema, as coordenadas podem ser obtidas com um teodolito; ao visar-se o astro com um teodolito nivelado, obtém-se $Z$ no limbo vertical do aparelho e, se o observador conhecer o meridiano, pode-se obter também o azimute do astro.

Observa-se que este sistema é tipicamente local, pois as coordenadas $A$ e $Z$ dependem da posição do observador, bem como a época em que a observação foi realizada, assim, este sistema variam no tempo e no espaço.

As coordenadas retilíneas neste sistema são:

$$
\begin{gathered}
x=\operatorname{sen} z \cos A \\
y=\operatorname{sen} z \operatorname{sen} A \\
z=\cos z
\end{gathered}
$$

\subsubsection{Sistema de Coordenadas Horárias}

Sistema geocêntrico;

Orientação: levogiro;

Plano fundamental é o equador;

Coordenadas:

\section{Eixos:}

$$
\begin{gathered}
H-\text { ângulo horário } \\
\delta-\text { declinação }
\end{gathered}
$$

$X$ : formado pela interseção do meridiano do observador com o equador celeste

$$
(+p / s u l)
$$

$Y$ : coincide com a linha leste - oeste;

$Z$ - coincide com o eixo de rotação da terra (ou esfera).

Figura 7. Sistema de Coordenadas Horárias

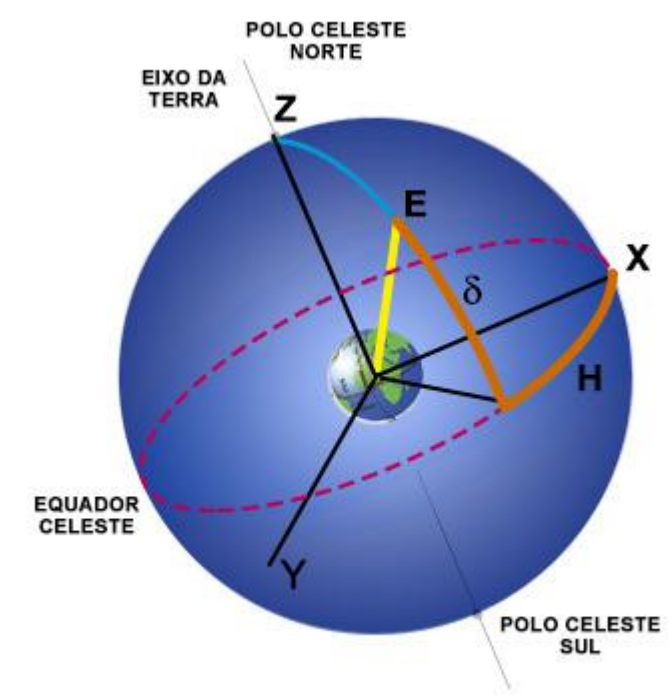

Fonte: Autor.

Neste sistema de coordenadas, tem: a abscissa esférica é o ângulo horário $\mathrm{H}$ é $\mathrm{O}$ arco de equador, delimitado pelo semi meridiano celeste do astro, a origem é o SMS e o sentido de contagem é retrógrado (por oeste). Varia de $0^{\circ} a 360^{\circ}$.

Devido sua vinculação com problemas horários, usualmente é expresso em hora, assim, variando de $0 h$ a $24 h$; a ordenada esférica é a declinação $\delta$ do astro, é definido como o arco do meridiano celeste do astro, contado a partir do equador até o astro. Varia de $0^{\circ}$ (astro no equador) $a \pm 90^{\circ}$

Convencionalmente positiva as declinações no hemisfério norte.

O sistema de coordenadas horárias é dito misto, pois a declinação $\delta$ do astro não depende da posição do observador, isto é, para qualquer observador um determinado astro terá a mesma declinação, se observado no mesmo instante físico. 
No entanto, o ângulo horário está depende do meridiano do observador, assim sendo, a abscissa $H$ depende da posição do observador.

As coordenadas retilíneas neste sistema são:

$$
\begin{gathered}
x=\cos \delta \cos H \\
y=\cos \delta \operatorname{sen} H \\
z=\operatorname{sen} \delta
\end{gathered}
$$

Considere o triângulo esférico $A B C$, onde $A$ é o zênite, $B$ é o astro a ser analisado e $C$ é o polo norte,

Figura 8. Triângulo de Posição



Fonte: O Autor.

Desta forma temos:

$$
\begin{gathered}
a=z, b=\left(90^{\circ}-\phi\right), \\
c=\left(90^{\circ}-\delta\right) \text { e } C=H .
\end{gathered}
$$

Aplicando a equação (1) do Teorema 1 no triângulo $A B C$, temos

$$
\begin{aligned}
\cos (z)=\cos & \left(90^{\circ}-\phi\right) \cos \left(90^{\circ}-\delta\right) \\
& +\operatorname{sen}\left(90^{\circ}-\phi\right) \operatorname{sen}\left(90^{\circ}\right. \\
& -\delta) \cos H,
\end{aligned}
$$

Assim, utilizando as identidades trigonométricas de seno e cosseno, obtemos:

$$
\begin{array}{r}
\cos (z)=\operatorname{sen}(\phi) \operatorname{sen}(\delta) \\
+\cos (\phi) \cos (\delta) \cos H \\
\cos (z)-\operatorname{sen}(\phi) \operatorname{sen}(\delta) \\
=\cos (\phi) \cos (\delta) \cos H
\end{array}
$$

$$
\begin{aligned}
\cos H= & \frac{\cos (z)}{\cos (\phi)}+\frac{\operatorname{sen}(\phi) \cdot \operatorname{sen}(\delta)}{\cos (\phi) \cdot \cos (\delta)} \\
\cos H & =\cos (z) \cdot \sec (\phi) \cdot \sec (\delta) \\
& -\operatorname{tg}(\phi) \cdot \operatorname{tg}(\delta)
\end{aligned}
$$

Ao nascer do Sol temos que o ângulo horário é $z=90^{\circ}$, pois o Sol se encontra no horizonte. De (1) temos:

$$
\cos H=-\operatorname{tg}(\phi) \operatorname{tg}(\delta)
$$

A equação (6) nos permite calcular quanto tempo o Sol permanece acima do horizonte em um certo local e em certa data do ano, pois o tempo de permanência acima do horizonte será duas vezes o ângulo horário desse astro no momento do nascer ou ocaso (FILHO, 2014).

\subsection{Aplicações}

\subsubsection{Distância entre dois pontos}

Em Geometria Plana, sabe-se que um triângulo possui seis elementos, três lados e três ângulos. E se conhecemos as medidas de três desses elementos, sendo pelo menos um deles um dos lados, podemos determinar todos os demais elementos do triângulo, bem como sua área. Para isso usa-se as relações conhecidas para triângulos no plano, como a lei dos senos e a lei dos cossenos (NETO, 2013). Mas se quisermos, por exemplo, conhecer as distâncias entre duas cidades separadas uma da outra por alguns milhares de quilômetros, mesmo conhecendo suas coordenadas geográficas e utilizando outra cidade como referência para obter um tri ângulo, ainda assim não teríamos a mesma precisão que temos quando trabalhamos sobre a esfera. As aplicações foram baseadas segundo Alves (2009).

Qual a distância entre a UFMS/CPTL (Universidade Federal de Mato Grosso do Sul- Campus Três Lagoas) e UNOESTE (Universidade do Oeste Paulista), sabendo que as latitudes e longitudes são $20^{\circ} 47^{\prime} 03.8^{\prime \prime} S, 51^{\circ} 39^{\prime} 56.3^{\prime \prime} W$

e

$$
22^{\circ} 07^{\prime} 02.3^{\prime \prime} S, 51^{\circ} 26^{\prime} 55.1^{\prime \prime} W
$$


Respectivamente (extraído de https://www.google.com.br/maps).

Para resolução da aplicação, chamaremos de $B$ a UFMS e de $C$ a UNOESTE, consideremos o ponto $A$ como sendo o polo Sul. Assim interligando os pontos, temos um triangulo esférico $A B C$.

Figura 9. Distância entre UFMS E UNOESTE

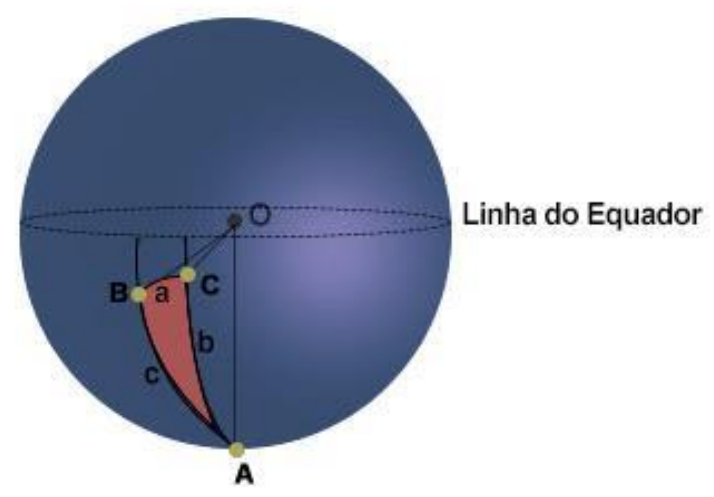

Fonte: O Autor.

Analisando o triangulo esférico, temos

$$
\begin{gathered}
b=90^{\circ}-20^{\circ} 47^{\prime} 03.8^{\prime \prime}=\left(69^{\circ} 12^{\prime} 56,2^{\prime \prime}\right) \\
=(69,21)^{\circ}, \\
\mathrm{e} \quad \\
\begin{array}{c}
c=90^{\circ}-22^{\circ} 07^{\prime} 02.3^{\prime \prime}=\left(67^{\circ} 52^{\prime} 57,7^{\prime \prime}\right) \\
=(67,89)^{\circ} .
\end{array}
\end{gathered}
$$

O ângulo $A$ é o ângulo formado pelos planos $B O A$ e $C O A$, ou seja, é a diferença entre as longitudes de $B$ e $C$ :

$$
\begin{gathered}
A=51^{\circ} 39^{\prime} 56 \cdot 3^{\prime \prime}-51^{\circ} 26^{\prime} 55 \cdot 1^{\prime \prime}=\left(16^{\prime} 2^{\prime \prime}\right) \\
=(0,27)^{\circ} .
\end{gathered}
$$

Para nossa aplicação consideramos a Terra uma perfeita esfera com raio de $6400 \mathrm{~km}$ e o centro denotado por $O$. Inicialmente, estudaremos o caso onde dois pontos $K$ e $L$ pertencem a uma circunferência máxima na esfera e ângulo $K O L=1^{\circ}$. Neste caso concluímos que

$$
d(K, L)=\frac{2 \pi 6400}{360} \mathrm{~km} \cong 111,7 \mathrm{~km}
$$

Quando dois pontos estão sobre uma circunferência máxima de centro $O, \quad$ a distância entre elas é a medida do ângulo KOL multiplicada por $111,7 \mathrm{~km}$.

Aplicando o teorema dos cossenos no triângulo esférico $A B C$, temos:

$$
\begin{aligned}
& \cos (a)=\cos 69,21 \cos 67,89 \\
&+\operatorname{sen} 69,21 \cdot \operatorname{sen} 67,89 \cdot \cos 0,27 \\
&=0.38 \\
& \text { Logo, } a \approx 1,17^{\circ} . \\
& \text { Como } B C \text { é um arco contido numa }
\end{aligned}
$$
circunferência máxima, então a distância entre $B$ e $C$ é $1,17^{\circ}$ multiplicado por $111,7 \mathrm{~km}$, ou seja, aproximadamente $131,15 \mathrm{~km}$.

\subsubsection{Tempo de Permanência do Sol}

Quanto tempo permanecerá o Sol acima do horizonte em Três Lagoas/MS, cuja latitude é $20^{\circ} 45^{\prime} 04^{\prime \prime} S$ em um dia de Solstício de verão no hemisfério sul, em que a declinação do Sol é $-23.43^{\circ}$ ? (Declinação obtida, segundo: Associação Nacional de Cruzeiros Entidade de Utilidade Pública.)

$$
\cos H=-\operatorname{tg}\left(-20^{\circ} 45^{\prime} 04^{\prime \prime}\right) \operatorname{tg}\left(-23.43^{\circ}\right),
$$

então,

$$
\cos H=-0,1642
$$

ou ainda, $H=99,45^{\circ}$. Portanto o tempo de permanência do sol é $2 H=198,90^{\circ}$. Como queremos saber o tempo de permanência do sol e sabendo que 24 horas é o tempo que o sol leva para dar uma volta sobre a terra, então:

$$
\begin{gathered}
360 \cdot t=2 H \cdot 24 \\
360 \cdot t=198,90^{\circ} \cdot 24 \\
t=13,26
\end{gathered}
$$

Portanto, o Sol ficará acima do horizonte aproximadamente 13,26 horas.

\section{DISCUSSÃO}

Para o estudo da geometria esférica aplicado na astronomia foi necessário um conhecimento abrangente de conceitos da 
geometria esférica e conceitos geográficos, como eixo, paralelos, meridianos e polos. Este estudo foi efetuado através de resolução de exercícios e seminários apresentados durante o desenvolvimento do trabalho, possibilitando verificar atuação da matemática em aplicações geográficas e astronômicas. Deste modo foram verificados teoremas, corolários perante a geometria esférica serem aplicados na astronomia.

\section{CONCLUSÃO}

Através do presente trabalho foi possível analisar o papel importante da matemática sendo aplicada na astronomia utilizando de conhecimentos geográficos. A partir de relações geométricas, com analises na esfera, no caso, o globo terrestre. Partindo de conceitos espaciais para aplicações práticas na própria astronomia. Sendo de grande importância e interesse, fazer essa união de temas, a fim de um único foco de aplicação, despertando o interesse para o aprendizado e contribuindo para a formação do aluno.

\section{REFERÊNCIAS}

ABREU, M. S. Geometria esférica e trigonometria esférica aplicadas à astronomia de posição. São João Del-Rei: PROFMAT, 2015.

ALVES, S. A Geometria do globo terrestre. Revista do Programa de Iniciação Cientifica OBMEP, Rio de Janeiro, 2009.

ARANA, J, M. Astronomia de posição: notas de aula. Presidente Prudente: Unesp, 2000.

Associação Nacional de Cruzeiros Entidade de Utilidade Pública. Disponível em: <https://www.ancruzeiros.pt/ancdrp/declinaçãodo-sol>. Acesso em: 10 ago. 2018.

FILHO, K. S. O.; SARAIVA, M. F. O. Astronomia e Astrofísica. Porto Alegre: Universidade Federal do Rio Grande do Sul, 2014.
NETO, A. C. M. Geometria. Rio de Janeiro: Sociedade Brasileira de Matemática, 2013. (Coleção PROFMAT).

PATAKI,I. Geometria esférica para formação de professores: uma proposta interdisciplinar, 2003. Dissertação (Mestrado em educação Matemática) - Pontifícia Universidade Católica de São Paulo, São Paulo, 2003

Editor chefe:

Dr.: Jair R. Garcia Jr. - UNOESTE

Editores:

Dr.: Robson Augusto Siscoutto - UNOESTE

Dra.: Ana Paula Marques Ramos - UNOESTE

Editor de layout:

Ana Carla Lopes Giroti - UNOESTE 